REFLEXIÓN

Recibido: $15 / 10 / 2013$

Revisado: 15/11/2013

Aprobado: 15/12/2013

\title{
LA EDUCACIÓN MUSICAL EN LATINOAMÉRICA: REFLEXIONES ENTRE LA ESTÉTICA DECOLONIAL Y ALTERMODERNA
}

\author{
MARIO FERNANDO EGAS VILLOTA \\ Universidad de Nariño \\ Grupo de investigación de educación y pedagogía GIDEP
}

\begin{abstract}
RESUMEN
Desde el original Sistema de Educación Musical surgido en Venezuela como proyecto social, son muchas las Redes que como eco de su exitoso desarrollo se implementan en varios países de América Latina. Ellas, sin embargo, enfrentan el reto de constituirse en una alternativa formativa para la potenciación de la sensibilidad humana desde la valoración de las características de los contextos sociales particulares como postura epistemológica que asume la ruptura no sólo de las precariedades económicas sino de la comprensión del arte musical como experiencia transformadora. De este modo surge la reapropiación de la aisthesis en tanto percepción sensible, en distanciamiento de una concepción clásica cercana a la estética de lo bello, que por otro lado implica la colonialidad manifiesta en la reproducción acrítica de principios educativos en el campo de la música, situados desde pilares como los repertorios, los instrumentos, las propuestas metodológicas de origen europeo, entre otros aspectos. Así mismo la pregunta sobre la formación como búsqueda o impulso de un devenir humano desde la emergencia de una nueva cultura altermoderna surgida en el mestizaje globalizado. Esta realidad convoca la reflexión de los programas de educación superior quienes afrontan el conflicto creciente por desintegrar los andamiajes de la tradición, enfocándose en la formación de un pensamiento creativo y una educación expresiva.
\end{abstract}

Palabras clave: Estética Decolonial, Estética Altermoderna, Musical Latinoamericano, Música, Experiencia Mística.

\begin{abstract}
Since the original System of Music Education emerged in Venezuela as a social project, many networks that echo its successful development are implemented in several countries in Latin America. They, however, face the challenge of becoming a formative alternative for the enhancement of human sensibility from the assessment of the characteristics of particular social contexts as an epistemological position that assumes the rupture not only of economic precarities but of the understanding of the Musical art as a transforming experience. In this way arises the reappropriation of aisthesis as a sensitive perception, distancing from a classical conception close to the
\end{abstract}


aesthetics of the beautiful, which on the other hand implies coloniality manifested in the uncritical reproduction of educational principles in the field of music, From pillars such as repertoires, instruments, methodological proposals of European origin, among other aspects. Also the question about the formation as search or impulse of a human becoming from the emergence of a new altermodern culture emerged in the globalized mestizaje. This reality summons the reflection of the higher education programs who face the growing conflict by disintegrating the scaffolding of tradition, focusing on the formation of creative thinking and expressive education.

Key words: Decolonial aesthetics, altermodern aesthetics, Latin American musical, music, mystical experience.

\section{Introducción}

Al hablar del arte musical Latinoamericano enfrentamos de entrada la dificultad de intentar sus definiciones partiendo del sentido implícito en los conceptos que lo delimitan cuyo origen corresponde a un marco preestablecido en las ideas de la cultura occidental inculcadas tras varios siglos de implementación de sus formas de realización ejecución y enseñanza. De este modo el primer escollo es la definición de la música como Arte que porta la aceptación, tácita, de sus dimensiones comunicativa y estética. Quiere esto decir que debemos asumir su discernimiento dentro de la discusión sobre la estética.

La filosofía y el Arte han planteado el final de la estética metafísica es decir la ruptura con las ideas de una estética de la trascendencia. Esta condición propia de las discusiones en la filosofía y en el arte cuestiona incluso el concepto de estética pues su conformación en una perspectiva histórica representa entre otras de sus características: la relación del arte con la naturaleza en tanto acto mimético, convirtiendo la obra en una búsqueda esencialista de una verdad, pues pretende alcanzar el lugar de lo bello, que corresponde a una condición metafísica, no a una propiedad de los objetos; así mismo su concepción como dimensión moral que se enfoca en el abandono de las pasiones; de otra parte la experiencia mística singularidad del cristianismo medieval el cual, además, acogió el doble legado racional y moral canónico clásico, que condena la "concupiscencia oculorum" o disfrute exacerbado del arte a través de lo observado; la desconfianza en la experiencia de los sentidos; el reconocimiento de la aisthesis que de todos modos plantea una cognitio sensitiva, al juntar la revelación de una verdad esencial y la sensibilidad; la desconfianza en que el arte pueda tocarse con la venerada racionalidad; la autonomía del lenguaje musical, cuya verdad se avizora más allá de la razón; la preeminencia apriorística de lo bello; la formación del gusto principio puramente subjetivo distinto a los juicios de conocimiento o morales; la fe en un futuro progreso sobre el pleno uso del albedrío humano, que promueve la actitud de no conformidad con una realidad sin esperanza; la resolución constante y positiva de la tensión entre la atracción de la realidad que atrapa y el impulso ético del hombre cuyo fin es la transformación de la sociedad; el sacrificio individual en beneficio del futuro feliz en una sociedad ideal ; el cumplimiento de la ley como principio moral en la educación de la totalidad huma- 
na ideal de oposición y lucha contra el egoísmo; la perspectiva de la obra de arte como creación abierta a la multiplicidad interpretativa.

En síntesis y sin pretender una visión exhaustiva del fenómeno, la estética implica entre otros aspectos: la postura gnoseológica que supone la dualidad sensibilidad-entendimiento; la experiencia mística que se apega a los conceptos de lo bello en tanto revelación por la vía moral y racional; la formación del gusto; la bifurcación de la expresión artística como verdad de la interioridad individual o mímesis de la grandiosidad de la naturaleza; la experiencia estética sinónimo de comprensión de la condición humana desde el reconocimiento de la acción intersubjetiva, cuya base es el papel fundante del lenguaje en toda comprensión, enfocando su lente hacia la doble idea de historicidad y universalidad del arte.

Como puede apreciarse el concepto de estética nacido en plena ilustración se constituye en un compendio de principios de las posturas de la modernidad. En el campo de la música convoca la discusión sobre su condición de lenguaje particular que eleva el espíritu, alcanza lo bello, permite la trascendencia, estimula la racionalidad o se desliza más allá de toda razón. Sin duda, alguna de estas es la forma como se ha comprendido y aceptado la condición de la música al menos en los últimos tres siglos. De dicho pensamiento son herederos los proyectos de educación musical en Latinoamérica los cuales no estuvieron exentos del deseo de beber de la fuente nutricia de la cultura europea implicando la acogida de medios expresivos como la orquesta y la preferencia por ciertos instrumentos, así como por géneros formas y estilos musicales. También los procesos educativos en el campo de la música ocurren de acuerdo al modelo europeo en relación con las técnicas, los métodos y aún el tipo de escuelas.

Walter Mignolo en una crítica a la modernidad en su doble discurso como relato salvífico -cuyo proceso requiere de la explotación, la represión, la deshumanización y el control de la población- y a la vez como colonialidad, es decir la colonización del imaginario de los dominados que actúa desde la interioridad de ese imaginario, plantea la operación cognitiva de colonización de la aesthesis por la estética. Aesthesis referida a "sensación", "proceso de percepción", "sensación visual", "sensación gustativa" o "sensación auditiva", el cual según Mignolo se restringe, y de ahí en adelante pasa a significar "sensación de lo bello", planteando la estética como teoría, y el concepto de arte como práctica. Si bien no es usual proponer el análisis de la estética y del arte en relación con la música los vínculos e interrelaciones de estas con la música llamada culta es clara.

En una perspectiva diferente Nicolás Bourriard director de la escuela superior de bellas artes en París y curador en exposiciones de arte contemporáneo lanza su "manifiesto altermoderno" cuyos principios expresan entre otras ideas que: el postmodernismo ha llegado a su fin; una nueva modernidad está emergiendo reconfigurada a una era de globalización entendida en sus aspectos económicos, políticos y culturales; la comunicación y emigración están afectando el modo en que vivimos; nuestra cotidianidad surge en un universo caótico y atiborrado; multiculturalismo e 
identidad están siendo desplazados por la creolización una especie de mezcla intercultural; los artistas ahora comienzan desde un estado globalizado de la cultura; los artistas están respondiendo a una nueva percepción globalizada; ellos cruzan un paisaje cultural saturado de signos, creando nuevos senderos con formatos de múltiple expresión y comunicación.

Esta postura constituye un nuevo panorama para comprender los fenómenos educativos en el campo de la música a partir de una base que implica la aceptación de una realidad dada sin entrar en cuestionamientos de los caracteres de origen histórico que han dado forma a las expresiones artísticas del presente. En este sentido con seguridad el avance de los medios tecnológicos constituye la puerta de entrada de todo estudio y comprensión del fenómeno.

\section{REDES Y SISTEMA}

En Venezuela, las ideas y el liderazgo en el campo de la pedagogía musical del maestro José Antonio Abreu, vinculado a las agrupaciones orquestales de perfil sinfónico y de música coral dio inicio en la década de los años ochenta, a una red de carácter nacional conformada por doscientas cincuenta mil personas aproximadamente. El "Sistema" busca una "revolución social sin paralelo" y la "democratización del arte musical". ${ }^{1}$ Se deposita la confianza en la pedagogía musical, como manera de superar: brechas sociales, desigualda-

1. FUNDACIÓN MUSICAL SIMÓN BOLÍVAR FUNDAMUSICALBOLIVAR. (2010). Filosofía. Recuperado de http://www.fesnojiv.gob.ve/ des, generando oportunidades laborales, vitales y consolidando un proyecto cultural sobre la afirmación de que "la música es un reflejo del alma de los pueblos"2.

Además se afirma:

El quehacer orquestal y coral [...] implica, necesariamente, la formación de un espíritu solidario y fraterno, un vigoroso desarrollo de la autoestima y el cultivo de los valores éticos y estéticos [...] la formación de la personalidad [...] despertar de la mente [...] desarrollo de la sensibilidad ${ }^{3}$.

En su ejecución, se "provee la capacitación laboral en la especialidad de construcción y reparación de instrumentos musicales, con el objetivo de afianzar una red nacional de microempresas fabricantes de instrumentos destinados al mercado nacional y latinoamericano"4.

Además, pretende extender sus bondades a la prevención de los conflictos sociales pues "contribuye a fomentar el uso adecuado del tiempo libre, manteniendo a los niños, niñas, adolescentes y jóvenes alejados de las drogas, el alcoholismo, la prostitución, la violencia y la delincuencia"s.

En las esferas: personal, familiar y comunitaria, se enfoca en el desarrollo moral, intelectual y espiritual; de igual modo en constituir, opciones económicas para superar las limitaciones del círculo familiar y promover propuestas creadoras de cultura y enriquecimiento de la interacción social, desde la conforma-

2. RUIZ, E. (2012). El Sistema Filosofía. Recuperado de http://edicsonruiz.com/es/elsistema.php

3. FUNDAMUSICALBOLIVAR Op. cit.

4. Ibíd.

5. Ibíd. 
ción de intérpretes y públicos sensibles a la música, en un espectro amplio que supere el dilema entre música popular y música académica ${ }^{6}$.

Como testimonio de la transformación y percepción comunitaria:

La música no queda excluida del ser $\mathrm{y}$ del quehacer diario, sino que nutre y es nutrida por esa cotidianidad, despertando su sentido estético, haciendo que sin posturas artificiales se cultiven la armonía y la belleza en diferentes espacios: la naturaleza, la ciudad y el propio individuo. Se aprende entonces a encontrar el arte, no solamente en los museos y conciertos, sino en los ambientes, en las personas y en las cosas de todos los días ${ }^{7}$.

Aún en la actualidad, dicho programa se desarrolla con altos reconocimientos a nivel mundial y ha sido acogido en más de veinticinco países: promueve la presentación de conciertos en importantes escenarios de Europa y América bajo la dirección de los más destacados directores de talla mundial, recibiendo elogios por su alta calidad interpretativa.

Es así como se ha replicado en nuestro país en proyectos análogos anclados al Plan Nacional de Música para la Convivencia que se articula en los campos de creación, memoria y diálogo cultural, a través de proyectos de emprendimiento y procesos de gestión mediante la participación social en torno a la música como:

Una oportunidad para tejer Nación desde la expresión musical [...] como factor de construcción de ciudadanía y de fomento a los valores, la creatividad artística, la cohesión social, el mejora-

6. CENTRO NACIONAL AUTÓNOMO DE CINEMATOGRAFÍA (Productor) \& ARÉVALO A. (Director) Tocar y luchar [Documental]. Cinema Sur Venezuela. Recuperado de http://www.youtube.com/ watch?v=OeTOSUhYlxs

7. FUNDAMUSICALBOLIVAR. Op. cit. miento de la calidad de vida y la búsqueda de la convivencia pacífica ${ }^{8}$.

En consecuencia la Alcaldía de Medellín crea el programa denominado Red de Escuelas de Música, cuyo propósito es generar y fortalecer procesos de convivencia y cultura ciudadana, mediante la formación a través de la música, en barrios y comunas. A su vez la Alcaldía de Pasto crea la Red de Escuelas Musicales bajo los principios de identidad, pertinencia, relevancia, complementariedad, transparencia $\mathrm{e}$ igualdad y desde la promoción de valores concernientes al desarrollo humano como la integración, equidad y concertación.

Su visión aspira a la inclusión social al crear:

Oportunidades de futuro [...] habilidades para el manejo pacífico de conflictos: percepción de límites, responsabilidad social y manejo de emociones, Perseverancia y disciplina [...] mayor dedicación a la Escuela de Música que a otras opciones de recreación [...] sujeción a marcos colectivos de bienestar9.

La Red, beneficia a personas entre los ocho y catorce años de edad, con características de marginación, pobreza y desplazamiento por la violencia.

\section{MÚSICA, ESTÉTICA Y EDUCACIÓN}

Los proyectos de Educación musical abarcan una compleja relación de la pedagogía musical con la filosofía y con el arte. Siendo claro el legado de la concepción moderna particularmen-

8. Ibíd.

9. Ibíd. 
te en relación a los ideales sintetizados en el pensamiento de Schiller, los cuales aspiran al ascenso humano para beneficio general de la sociedad, desde el abandono de los logros individuales. En este contexto, la educación se orienta en la formación estética: ofreciendo la posibilidad de una libertad trascendental, una libertad de elección y se inclina por la disposición individual hacia la realización de los más altos logros humanos.

De otra parte, la comprensión de la educación como proceso permanente y acontecimiento que desborda el límite institucional, mecanismo de transformación ó incorporación de una fuerza interior para el fluir vital constante, potencia las acciones cotidianas, validando así la pedagogía como formación para la interacción ${ }^{10}$, la convivencia y la construcción de la vida. En este sentido, una postura pedagógica no ocurre en un espacio de abstracción sino en un lugar específico delimitado por las condiciones culturales, sociales y en general, por las interrelaciones de los grupos humanos y sus formas de comprender el mundo e interactuar en él. Admitamos que es clara la imbricación de aspectos pedagógicos, éticos y estéticos.

Todo lo anterior ocurre en la cercanía de una concepción estética cuya acción sucede sin necesidad de un impulso exterior de coacción pues es evidente la profundidad del enraizamiento o la afectación de los imaginarios que dan por hecho un ideal de formación pro-

10. GÓMEZ, G.J. (2007). "Representaciones sociales de la práctica pedagógica del docente universitario en la formación de licenciados en Artes Plásticas y/o Visuales: concepciones e incidencias". Tesis Doctoral. Universidad del Cauca -Rudecolombia- Popayán, Cauca pio de la modernidad. Este marco encuentra en la orquesta sinfónica el lugar ideal de formación de la pretendida experiencia estética. La orquesta como lugar de encuentros, escenario de primer orden e institución formadora, exige: disciplina, esfuerzo individual, silencio, disposición al trabajo en equipo, repertorio de música de los grandes maestros, instrumentos musicales específicos, procesos usualmente tenidos en alta estima en este campo como la lectura de partituras. Todo esto, en correspondencia con la creencia del valor en sí de la música llamada "culta" o clásica" -entre otras denominaciones-, convertidos en principios tácitos o declarados.

Tradicionalmente, bajo la norma disciplinar subyace la aceptación de la condición de discípulo, esto es, de un sujeto cuyo largo camino de carencias debe subsanarse a partir de un modelo imitativo, provisto por el maestro, en correspondencia con la repetición de esquemas técnicos, -tanto para las formas interpretativas instrumentales, como para las prácticas conductuales-, incidiendo en el desarrollo de la libertad creativa.

El estudio de un instrumento sinfónico implica la consideración de su significación singular: su forma, sus materiales, su sonoridad. Traduce formas estéticas, desde el umbral de lenguajes que relacionan historicidades $\mathrm{y}$ nos ofrecen diversidad de sentires y formas prácticas de abordar el mundo. En vista de esto, AHARONIÁN (2012) afirma el protagonismo de los instrumentos musicales en los conflictos de colonización, al relatar: "los tatarabuelos indígenas habían reaccionado a la conquista apropiándose del violín -una 
de las armas de los conquistadores- e integrarlo a su modo particular de ver el mundo. Los tíos mestizos habían redondeado la apropiación"11. Quizás una de las preocupaciones importantes en la pedagogía musical Latinoamericana, corresponde a la imposición de expresiones foráneas cohibiendo las riquísimas expresiones musicales propias, debido a los siglos de dominación cultural y al uso de los canales contemporáneos de difusión de la música capaces de moldear los gustos de masas consumidoras. Es claro que los procesos formativos de los proyectos orquestales juveniles para la región comportan la posibilidad de una experiencia singular, que si bien no resuelve males generales como la injusticia social, la marginación, la pobreza total, por encima de la crítica ideológica, permite una comprensión menos próxima al desahucio del modelo colonialista. Este último, pareciera postular la división binaria de fuerzas en contienda ó el fenómeno estético como hecho individual, en procura del alivio de la nefasta masificación producida por la acción colonialista. En cualquier caso, la defensa de la llamada mesomúsica ${ }^{12}$, denominación de la música popular, no olvida que los componentes rítmicos, armónicos, melódicos y expresivos apreciados como caudal de la riqueza propia en innumerables géneros musicales, llegaron con la cultura ajena. En este sentido la Red cuenta con un centro de música tradicional que en sus lineamientos pedagógicos deberá

11. AHARONIÁN, C. (2012) La enseñanza de la música y nuestras realidades. Revista (Pensamiento), (palabra) y obra, 7 artículo 2. Universidad pedagógica Nacional. Extraído el 20 de agosto, 2012 de:http:// www.pedagogica.edu.co/revistas/ojs/index.php/ revistafba/search/results

12. ARETZ, Isabel. América Latina en su música. $8^{\mathrm{a}}$ Edición. México: Siglo Veintiuno, 1997, p. 39. definir su posición frente a múltiples y complejos aspectos como el rescate de un pasado siempre en discusión, la definición y estudio de los instrumentos tradicionales, el estudio de sus técnicas interpretativas y la definición de sus repertorios.

En visión retrospectiva, resulta singular la condición del contexto educativo, signado por una rica tradición teologal, reconocida según Zúñiga (1999), a la cultura pastusa ${ }^{13}$. Este panorama, nos conduce a la reflexión de la idea de la formación, cuyo estudio en Gadamer (2007) rescata la condición original del término, refiriéndolo a la "mística medieval", a la reconstrucción en sí del alma de Dios; según la cual se concibe la Creación ${ }^{14}$. Esta visión, coherente con un modelo estético místico, se aviene de modo conveniente a la comprensión del proceso pedagógico musical local. En la línea de la implementación, la experiencia estética de la Red de Escuelas de Música no se limita a su relación con el encuentro de la verdad intrínseca, inherente a lo sonoro musical que algunos asumen como un lenguaje en sí mismo: de Schloezer y Scriabine (1990) -expresión de una verdad musical propia, que no encarna nada exterior, sólo su ser inmanente-15: sobrepasa los límites, provocando el trastorno, en tanto experiencia estética, de las sensibilidades y los espacios de develamiento. A su vez, se anuncia como esperanza para la redención del

13. ZÚÑIGA ERAZO, E. (2.002). Nariño, Cultura e Ideología. San Juan de Pasto: Universidad de Nariño. 449 p.

14. GADAMER, Op. cit., p. 38.

15. DE SCHLOEZER, Boris y SCRIABINE, Marina. Problemas de la música moderna. Barcelona: Seix Barral. 1973. $223 \mathrm{p}$. 
alma, aspiración que también motiva la práctica musical.

Frente a esta evolución de las ideas sobre la estética, la mayor inquietud parece surgir de la comprensión del ser en una dimensión divergente de la metafísica. Por ello el vínculo ético de la pedagogía con la formación se hermana de modo indudable en el propósito de convertirse en una acción sincera y transparente de acompañar la transformación permanente del Ser que se comprende como posibilidad constante más que realidad pre-constituida. En ello se juega el sentido pedagógico relacionado con las artes, cuya mirada se orienta hacia la educación musical: ahora cuestionamiento de lo instrumental para asignar un sentido total al despliegue de los potenciales humanos. En este sentido la expresividad, huella del empleo autónomo de la emotividad humana, fecunda la búsqueda de una educación a lo largo de la vida que encuentra en la música un área llena de riquezas vitales. En consecuencia es en el campo de la Educación musical en donde la visión crítica puede permitir la comprensión de la estética como resultado de las realizaciones humanas sobre la realidad más que en el misterio insondable de lo metafísico.

\section{REFLEXIONES FINALES}

El rico e importante caudal de expresiones musicales aporta a la revaloración y rescate del sujeto en sus precariedades por ello aflora de manera generosa la musicalidad. Entonces la música permite la cohesión e identidad de los jóvenes. La música adecuada a la tradición teológica se constituye en un acicate moral que confiere sentido a una participación colectiva copiosa en sus experiencias constituyendo otra forma de identidad.

En la implementación de la idea altermoderna, la incidencia de los medios tecnológicos en la formación del gusto, debido a una visión desesperanzada, que anuncia el declive de las posibilidades del ser, en un atrapamiento sin salida del efecto deformador de la televisión, el internet, el disco, la radio, el cine, entre otros, el reconocimiento del fenómeno musical como experiencia estética performativa que involucra al sujeto no sólo en su papel receptivo como oyente, sino productivo como intérprete hace de la obra de arte un proceso de interdependencia que permite el reconocimiento del papel activo del sujeto y asigna a los medios tecnológicos el rol de mediadores de la formación y de la construcción de un tejido simbólico constituido en la riqueza de las posibilidades vitales y en este sentido en disposición estética o impulso de renovación constante de un decurso ético. En la percepción altermoderna sólo la existencia de los medios tecnológicos ha posibilitado el surgimiento de géneros y propuestas musicales que serían imposibles sin el concurso de sus avances y recursos. Los intentos individuales promueven la solución creativa de la ruptura con el modelo estandarizado de educación a través del abandono del entorno escolar hacia nuevas ofertas de educación virtual que permiten más tiempo para dedicarse al estudio de la música.

Como posibilidad decolonial la expresión musical permite la manifestación de nuevas posturas y visiones del mundo a partir de la búsqueda del reco- 
nocimiento universal de una expresión y una cultura local, como reafirmación de la sensibilidad y la memoria.

Mientras las grandes industrias del disco pretenden continuar el monopolio de su mega industria imponiendo la idea de una nociva piratería de bajo perfil moral, como estrategia de protección y defensa de sus condiciones económicas más bien limitadas, los músicos encuentran en la piratería el mecanismo más eficaz de protección de su imagen, de difusión de su obra y de promoción de sus conciertos que les brinda un sustento económico interesante pues la venta de discos lo ubican en el último y restringido lugar de beneficiarios de los recursos que de otra parte para las disqueras son enormes y jugosos.

Las escuelas de formación musical de nivel universitario, llámense conservatorios, licenciaturas en música u otros programas de profesionalización deben revaluar su tarea en varios aspectos a saber: Apertura a la realidad musical desde la investigación en una proyección más clara hacia lo social; disposición de sus pilares formativos apoyados en maneras tradicionales como la enseñanza de la lectoescritura musical. 


\section{BIBLIOGRAFÍA}

AHARONIÁN, C. (2012). La enseñanza de la música y nuestras realidades. Revista (Pensamiento), (palabra) y obra, 7 artículo 2. Universidad pedagógica Nacional. Extraído el 20 de agosto, 2012 de: http://www.pedagogica.edu.co/revistas/ojs/index.php/revistafba/search/results

ALCALDÍA DE PASTO (2012). Red de Escuelas de Formación Musical Recuperado de http://www.musical. pasto.gov.co/

ARETZ, Isabel (1997). América Latina en su música. $8^{a}$ Edición. México: Siglo Veintiuno.

BAYER, R. (1975). Historia de la estética. México: Fondo de Cultura Económica.

CAJIAO, F. (2009). La educación artística. Revista (Pensamiento), (palabra) y obra. Universidad pedagógica Nacional. Recuperado de: http://www.pedagogica.edu.co/revistas/ojs/index.php/revistafba/search/results

CAPDEVILA, P. (2007). Historicidad y estructura de la experiencia estética. Una propuesta desde Hans-Robert Jauss. Recuperado de: $\Delta a i^{\prime} \mu \omega v$. Revista de Filosofía, 42, 127-144.

CENTRO NACIONAL AUTÓNOMO DE CINEMATOGRAFÍA (Productor) \& ARÉVALO A. (Director) Tocar y luchar [Documental]. Cinema Sur Venezuela. Recuperado de http://www.youtube.com/watch?v=OeTOSUhYlxs

COLOMBIA, MINISTERIO DE CULTURA (2008). Plan Nacional de Música para la Convivencia. Recuperado de http://gcn.mincultura.gov.co/el-festival/plan-nacional-de-música-para-la-convivencia-pnmc/).

DE SCHLOEZER, B. y SCRIABINE, M. (1973). Problemas de la música moderna. Barcelona: Seix Barral. 223 p.

FUBINI, E. (1971). La estética musical del s. XVIII a nuestros días. Barcelona: Barral Editores.

FUNDACIÓN MUSICAL SIMÓN BOLÍVAR FUNDAMUSICALBOLIVAR (2010). Filosofía. Recuperado de http:// www.fesnojiv.gob.ve/

GADAMER, H. (2007). Verdad y método. Salamanca: Ediciones Sígueme.

GUTIÉRREZ, C. (2002). Temas de filosofía hermenéutica: Conferencias y ensayos. Bogotá: Universidad Nacional de Colombia.

HANZA, K. (2008). La Estética de Kant: el arte en el ámbito de lo público. Pontificia Universidad Católica del Perú khanza@pucp.edu.pe Revista de Filosofía, 64, 49-63.

JAUSS, H. (1986). Experiencia estética y Hermenéutica literaria. Ensayos en el campo de la experiencia estética. Madrid: Taurus Ediciones.

KANT, E. (1876). “Crítica del juicio" seguida de las observaciones sobre el asentimiento de "lo bello y lo sublime" Carece de ciudad: Imp. de Federico Escamez y Centeno.

LÓPEZ SÁENZ, M. (2008) Arte como conocimiento en la estética hermenéutica. Endoxa: Series filosóficas, 10, 1998. Madrid: UNED.

MÓDULO DEL SISTEMA NACIONAL DE ORQUESTAS Y COROS JUVENILES E INFANTILES DE VENEZUELA en el Colegio San José de Calasanz. Recuperado de http://www.itakaescolapios.org/sedes\%20y\%20presencias/ venezuela/proyectos-y-presencia/caracas/modulo-del-sistema-nacional-de-orquestas-y-coros-juveniles-einfantiles/resumen-del-proyecto/preview_popup/file

RUIZ, E. (2012). El Sistema Filosofía. Recuperado de http://edicsonruiz.com/es/elsistema.php

SCHILLER, F. (1990). Kallias; Cartas sobre la educación estética del hombre; estudio introductorio de Jaime Feijóo - edición bilingüe. Barcelona: Anthropos.

ZÚÑIGA ERAZO, E., (2002). Nariño, cultura e ideología. San Juan de Pasto: Universidad de Nariño.

DE SCHLOEZER, Boris y SCRIABINE, Marina (1973). Problemas de la música moderna. Barcelona: Seix Barral. 